\title{
Le compromis autofictif
}

\author{
Emmanuel Samé \\ Université de Dijon
}

Que dit l'autofiction que ne disent ni l'autobiographie ni le roman? À partir de Fils (1977) de Doubrovsky, du Miroir qui revient (1984) de Robbe-Grillet et de À l'Ami qui ne m'a pas sauvé la vie (1990) de Guibert, nous tenterons, à travers une perspective littéraire et psychanalytique, de définir l'autofiction comme un compromis. D'une part, aux avatars parricides de Don Juan, Valmont ou Sade convoqués par l'autofictionnaliste fait écho la figure paternelle de l'analyste tentant de percer à jour la psyché de l'auteur. D'autre part, s'engageant en son nom propre comme auteur et comme narrateur, l'autofictionnaliste entre dans le genre autobiographique et s'engage consciemment, par l'homonymat auteur-narrateur-personnage, 
à parler symboliquement au nom du père cependant que, par l'entrée dans le pacte romanesque, il refuse de soumettre sa parole à tout regard comme à toute loi, supposant qu'elle demeurera ainsi authentique. Dans cette logique, il est compréhensible que Philippe Lejeune, par cette exigence de signification qui est le principe premier et positif de la quête autobiographique, devienne un avatar essentialiste et déterministe de la figure du psychanalyste et l'autobiographe, un analysant. La question du genre, dans l'autofiction, est donc celle, politique et érotique, de la résistance du fils à une position œdipienne devenue celle d'un autobiographe-analysant. Comment l'autofictionnaliste construit-il la figure donjuanesque d'un ultra-autobiographe? En quoi le pacte romanesque, justifiant l'illégalité autant que l'authenticité du propos de cet ultra-autobiographe, contribue-t-il à rebours à construire une écriture du secret ? Enfin, comment définir le défi donjuanesque de l'autofictionnaliste au père-autobiographe comme un compromis?

\section{L'ultra-autobiographe}

La figure de Don Juan, dont la posture libertine ne tient pas que du simple libertinage, se veut une revendication philosophique contre tout obscurantisme religieux. C'est en ces termes que Robbe-Grillet l'évoque dans Le Miroir qui revient; il est celui «qui a choisi sa propre parole, aventureuse, changeante et contradictoire, comme unique fondement de sa vérité, sa vérité à lui - humaine - qui ne peut exister que dans l'instant contre la Vérité de Dieu qui est par définition éternelle » (1984, p. 74). Le psychanalyste (et le médecin) apparaît comme cet homme 
du dogme et de la vérité éternelle cherchant à imposer une vérité figée à laquelle l'autofictionnaliste oppose une vérité glissante. Les écrits freudiens deviennent semblables à une Bible, Freud lui-même, à Dieu le père, les analystes, à de zélés officiants, et les patients à d'aveugles croyants désirant s'attirer le pardon des péchés en voulant bien reconnaître leurs fautes. La plume de l'autofictionnaliste, "pénis impénitent [qui] explose en gerbes de vocables » (Doubrovsky, 1977, p. 480), appartient à celui qui, connotation religieuse oblige, refuse la pénitence, c'est-à-dire la castration. Il est, référence faite au juif errant, ce «jouis errant» (ibid., p. 476) dont la «condition diasporique» (Doubrovsky, 2006, p.49) semble bien hystérique. Songeons à M. Klein, ce Juif hongrois de vingt-trois ans que Charcot, s'intéressant à l'hystérie masculine, présenta comme un véritable descendant d'Ahasvérus ou de Catarpilus, un voyageur névropathe mû constamment par un besoin irrésistible de se déplacer, de voyager, sans pouvoir se fixer nulle part et dont $\mathrm{H}$. Meige, un élève de Charcot, rend compte dans Le Juif errant à la Salpêtrière : étude sur certains voyageurs névropathes (1893). "Hystérique [Doubrovsky] converti[t] à tour de bras, à tour de rôle» (Doubrovsky, 1977, p. 190), adoptant tous les symptômes en sorte, irréductible, d'échapper sans cesse à l'analyste. Erratisme que, sur le plan biologique, le virus du sida, stigmate d'une homosexualité diabolique, ne cesse de mimer. «Retrovir, vade retro Satanas » (Guibert, 1990, p. 243), s'exclame ironiquement le narrateur de $\grave{A}$ l'ami qui ne m'a pas sauvé la vie, singeant l'exorcisme. Le corps du texte autofictif est soumis à une procédure d'examen par le censeur lejeunien comme le corps l'est par le médecin et la psyché par le psychanalyste. Ils sont en quête d'une maladie de genre. 
À s'illustrer de manière feinte en vertueux « catéchiste ", Robbe-Grillet, ce «bon fils » (Robbe-Grillet, 1984, p. 47) ironise dans Le Miroir qui revient sur cette " sensible tendresse filiale » (ibid., p. 57) et sur cette écriture qui, irrésistiblement, glisse vers l'autobiographie. La phrase liminaire - «J'ai longtemps cru, étant enfant, que je n'aimais pas la mer» (ibid., p. 13) -

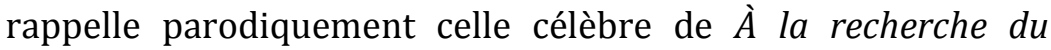
temps perdu : «Longtemps, je me suis couché de bonne heure » (Proust, 1913, p.13). De même que ce style autobiographique, fait de "volutes stylistiques», d' «acrobaties verbales», de "masques » et de "fioritures», est aux yeux de Doubrovsky l'équivalent de « feux d'artifice » (1989, p. 369), c'est-à-dire une écriture factice tendant à mettre en valeur son sujet de la manière la plus pompeuse, à la relecture du souvenir, le narrateur du Miroir qui revient est pris d'une grande envie de rire tant il lui semble falsifier son existence pour en faire un « objet bien sage, conform[e] aux canons du regretté Figaro littéraire : logique, ému, plastifié » (Robbe-Grillet, 1984, p. 17). Il reproche à son écriture «l'arrogance » (ibid.) des détails en trop petit nombre, « une maigre douzaine, isolés chacun sur un piédestal, coulés dans le bronze d'une narration [...] conforme justement à la pesanteur idéologique » (ibid.) d'une élite de droite. La mémoire défaillant, le grand-père de Robbe-Grillet laisse en « une constellation rassurante, claire, univoque » (ibid., p. 27) l'image officielle d'un homme qui a sacrifié sa vie à la cause de l'État à l'aune de ces photographies de famille qui, pour Guibert, donnent à voir « une espèce d'histoire digne, aplanie, interchangeable» (1981, p. 36), une histoire «bien colmatée et cohérente, sans failles » (ibid., p. 38), dans laquelle rien ne peut se déceler qui ne se sache déjà. 
L'écriture du souvenir tient de la dictée et de l'exercice scolaire. Tentation de l'ordre qui, pour Robbe-Grillet, relève de l'expérience autobiographique comme de l'expérience politique. L'évocation du nazisme et du stalinisme illustre jusqu'à quelle extrémité peut aller la sujétion du fils au père (Robbe-Grillet, 1984, p. 131-132). La découverte de la barbarie concentrationnaire marque un tournant décisif dans l'écriture de soi. Soudain, par la Libération (ibid., p. 165), vérité et liberté s'opposent. L'entreprise autobiographique s'apparente à une menace, un déterminisme dangereux dont il faut se libérer comme il faut se libérer, après les barbaries du XX $\mathrm{X}^{\mathrm{e}}$ siècle, de ce « dogmatisme [qui] n'est rien d'autre que le discours serein de la vérité (sûre de soi-même, pleine et sans partage) » (ibid., p.64). Pareille comparaison tend à démontrer comment l'autobiographe peut de guerre lasse être «menacé par cette pente, ce précipice qu'[il] côtoie» (ibid., p.58) et dont résolument il ne suffit pas de percevoir les dangers pour échapper à la «fascination" (ibid.), s'abandonner à « rassembler toute une existence vécue (qui, dans l'instant, faisait eau de toute part) en un volume clos, sans manques et sans bavures » (ibid.). L'autobiographe, en récitant, est dans une soumission au discours de l'autre comparable à une manière d'idiotie et, semblablement, d'inconscience. Le rapport du sujet au père comme au sens, à l'aune d'une religion, est évoqué par Sartre dans "L'enfance d'un chef» comme un mode factice d'apaisement. Lucien se croit délivré de ses inquiétudes et se jette sur la psychanalyse avec avidité se disant qu'il n'est plus besoin d'être en quête de lui-même (1939, p. 183). Berliac et lui ressemblent à deux écoliers tentant d'appliquer coûte que coûte la vérité psychanalytique et ses postures. Ils sont des «types foutus, des ratés» (ibid., p. 185). Au même titre que quelques 
papillons naturalisés, épinglés comme délinquants, leur nature semble à tout jamais inscrite. Irrécusables, ils n'ont plus à chercher qui ils sont. Ainsi, il en est pour Sartre comme il en serait pour l'autofictionnaliste : recourir à l'inconscient est une excuse pour expliquer sa mauvaise foi (Sartre, 1943, p. 87) et une manière, en somme, d'abandonner lâchement tout effort d'authenticité. Elle dispense de l'angoisse mais aussi de la liberté de se définir, montrant un individu pris dans un déterminisme défaitiste. Elle révèle et dérobe à la fois, met sous séquestre son enfance, son identité, impose ses analyses, ses complexes, une grille de lecture à travers laquelle l'individu n'est plus qu'un CEdipe (Doubrovsky, 1989, p. 389). Le « complexe de derrière les fagots » ou les « complexes maison » (Sartre, 1939, p. 183) évoqués dans Le Mur valent ironiquement « les grands concepts bateau» (Doubrovsky, 1977, p. 191) ou "[l]es schémas chouettes» (ibid., p. 191) de Fils. Le sujet, s'attribuant telle ou telle névrose et tel ou tel complexe, appartient très vite au « Musée imaginaire » (ibid, p. 190).

Face à Akeret, psychanalyste Suisse allemand, décrit sous les traits d'un aryen (Poirier, 2001, p. 92) poursuivant de son regard inquisiteur le narrateur de Fils et au professeur Van de Reeves, ex-nazi réfugié en Amérique du Sud dans Les Derniers jours de Corinthe, comme face à ce médecin au "physique de sadique de film de nazis» (Guibert, 1991, p.69) dans Le Protocole compassionnel, il ne reste plus à l'autofictionnaliste qu'à prendre en résistant le maquis et à se faire, tel Don Juan, «jouis errant» (Doubrovsky, 1977, p.476). En rivalité avec l'autobiographe-analysant, il décide, en se retranchant plus avant dans le roman, de reconquérir un regard sur soi. L'analysant se retournant sur son analyste, voici qui nous rappelle cette scène fameuse de «L'homme au magnétophone » 
(Sartre, 1969). Sur le plan de la critique littéraire, la scène trouve en effet à se rejouer dans la posture dissidente exprimée par Doubrovsky sur sa quatrième de couverture, position prise dans la célèbre lettre du 17 octobre 1977 adressée à Lejeune et citée par le critique dans son chapitre «Autobiographie, roman et nom propre » (1986, p. 63). Prenant pour complice et pour témoin le lecteur, il opère ce retournement par lequel, soudain, il portera un regard sur la figure d'un censeur lejeunien comme sur un psychanalyste sans qu'il soit donné à ces derniers de porter un regard sur lui en sorte que l'autofiction demeure ce «lieu de la parole qui [leur] échappe » (Darrieussecq, 1996, p. 378). Il pervertit - au sens étymologique de mettre sens dessus dessous - le procès autobiographique. À travers un parallèle entre, d'une part, perversion et sémitisme et, d'autre part, psychanalyse et nazisme, Doubrovsky, opposant au cœur de l'autofiction la figure d'un anal-yste à un « jouis errant » qui n'écrit que dans la crainte de «l'empallemand » (Doubrovsky, 1977, p. 321), fait glisser de manière tout à fait sartrienne la question de l'OEdipe du plan symbolique au plan politique mais sur les terres de l'autobiographie. L'autofictionnaliste, dans cette «terreur [barthésienne] de voir son moi se coaguler» (Robbe-Grillet, 2001, p. 191), fait l'économie d'un regard «par derrière » lejeunien qui le déposséderait en élaborant, comme l'écrit Robbe-Grillet, une «autobiographie consciente» et, ajoute-t-il, «consciente de son inconscience» (1994, p.17), mais en ce sens sartrien d'un refus de l'inconscient pressenti comme un déterminisme scientiste réduisant l'homme à un objet. Lejeune, affirmant par «science dogmatique» que «l'exigence de signification » est « ce principe positif et premier [...] de la quête autobiographique » (ibid., p.67), devient un avatar déterministe et dix-neuvièmiste de la figure de l'anal- 
yste susceptible, en prenant ce Roquentin par derrière, de le réifier en sorte que cette autobiographie et cette psychanalyse essentialistes se confondent. Ce discours ultra-analytique, par son entrée dans le pacte romanesque, soutient aux yeux du lecteur que l'auto-analyse autofictionnelle va au-delà de la psychanalyse tant qu'insoumise, elle évite, contrairement à l'autobiographe-analysant, de s'ancrer dans un genre (aux deux sens du terme que l'anglais précise à travers les formes lexicales distinctes gender et genre) comme de se figer en une posture œdipienne de la même manière que Don Juan, pour conquérir sa proie, fait valoir sa promesse amoureuse contre la promesse matrimoniale faite au père ou au mari.

\section{Le secret}

Le narrateur ferait émerger l'authenticité de son être à travers une promesse ultra-autobiographique associant l'illégalité de son propos à son degré de vérité. La psychanalyse, pour Doubrovsky et Robbe-Grillet ainsi que la médecine pour Guibert, à travers ces figures davantage sadiennes et totalitaires que réalistes, servent à l'instauration de cette dramatique politique. Toute la culture psychanalytique et médicale de nos narrateurs est mise à contribution. À l'aune de la promesse donjuanesque, il faut, pour que soient mesurées toute l'authenticité et toute la singularité d'un aveu, traduire son caractère exceptionnel et impérieux. Les données chiffrées des examens médicaux, les détails précis des effets du sida ou de son traitement dans l'œuvre de Guibert, toute la rhétorique psychanalytique, ses références bibliographiques freudiennes 
ou lacaniennes, les références raciniennes de Doubrovsky propres à l'hystérie de Phèdre ou d'Hippolyte, les éléments du Nouveau Roman intégrés à la Nouvelle Autobiographie de Robbe-Grillet, ses confessions sadiennes sont autant d'opérateurs qui instaurent une dramatique politique révélant un procès autobiographique désignant un inquisiteur et un hérétique. C'est là, tout en même temps, donner un statut légitime au couple du narrateur et du lecteur et ancrer toute la stratégie de la promesse ultra-autobiographique à la fois vitale, impérieuse et, ce faisant, censément authentique dans le cadre de l'indécidable.

La mort fait «acte d'authentification, elle pose un cachet de foi » (Guibert, 2001, p. 176). La dramatique politique du malade du sida «dénudé et exposé comme le corps dévêtu qui doit traverser le cauchemar. [Le] sang démasqué » (Guibert, 1990, p.14), laissent penser à ce juif poursuivi par les nazis comme l'atteste la référence à "Nuit et brouillard, le documentaire sur les camps de concentration » (ibid.). Toute cette dramatique se poursuivra au fil de l'œuvre. Elle initie la stratégie de la promesse ultra-autobiographique, car dès le déploiement de celle-ci, l'auteur évoque quelques lignes après le «silence», le «mensonge » face au " pli de la vérité » (ibid., p. 15). L'« aveu » devient ce danger, face aux figures de la loi, «à ce que le monde entier [lui] chie au même moment sur la gueule» (ibid., p. 16). Le niveau de langue utilisé sert évidemment à confirmer le caractère éruptif et spontané du propos, à l'image de toute la rhétorique de Doubrovsky quant à l'utilisation d'une langue inspirée de l'association libre: la naturalité du propos semble confirmer que le narrateur indexe sa langue à sa propre nature. Parlant familièrement, Guibert nous considérerait comme il le ferait d'un compagnon. La 
dramatique posée, la stratégie de la promesse ultraautobiographique peut agir pleinement: il faut donner au lecteur l'impression d'entrer en résistance, d'accueillir ce «juif» en temps de guerre et d'écouter là une confession nécessairement authentique qui lui sera un dictame. Le cadre hystérique de l'indécidable fera ensuite son effet.

De la même manière que Guibert, dans À l'ami qui ne m'a pas sauvé la vie, évoque cette dramatique politique du malade du sida, ce «juif» face aux forces médicales, patriarcales et sociales comparables aux nazis, Doubrovsky se constitue comme un névrosé, «jouis errant » (1977, p. 476) face à l'analyste nazi. Quant à Robbe-Grillet, c'est contre lui-même qu'il se défend ainsi qu'il l'annonce par ce " "Robbe-Grillet par luimême" ». C'est contre " [l]'idéologie toujours masquée, [cette] hydre-miroir, dont la tête coupée reparaît bien vite à neuf, présentant à l'adversaire son propre visage, qui se croyait vainqueur » (1984, p. 11) qu'il se bat. Ainsi va-t-il «emprunter la dépouille du monstre: voir par ses yeux, entendre par les trous de ses oreilles, et parler par sa bouche » (ibid.). Se méfiant de son propre "catéchisme », décidé à " [se] regarder de côté » (ibid., p.12), il appliquera le principe du Nouveau Roman, «ferment de liberté, de découverte» (ibid, p. 11), écriture semblable à «une activité dont la mobilité sans cesse lui échappe » (ibid., p. 12), à cette Nouvelle Autobiographie qu'il s'apprête à écrire. Dans cette dramatique, l'« explication définitive» (ibid., p.13) ou «véridique (celle, recueillie à la source, que fournit l'auteur lui-même!)» (ibid.), le «fonctionnement authentifié » (ibid.) ou la «signification réelle " (ibid.) sont ce danger à éviter de sorte que le lecteur suivra le narrateur non comme un "maître à penser » (ibid.) mais comme «un compagnon de route, d'invention ou 
d'aléatoire recherche» (ibid.) participant en quelque sorte au tumulte et à l'incertitude d'une création dont le vacillement, les détours, les digressions ou les régressions seront vécus comme un gage de spontanéité et d'authenticité.

Très vite, la figure du "psychanalyste amateur » (ibid., p.15) fait son apparition, constituant le couple lecteurnarrateur. Cependant que les romans robbe-grillétiens, à l'inverse du mythe psychanalytique, refusent le visible comme correspondance de l'invisible, Robbe-Grillet, dans sa Nouvelle Autobiographie, annonce une écriture «consciente de son inconscience» (1994, p.17), de sorte qu'à travers les Romanesques s'institue un théâtre reprenant en écho d'autres œuvres de l'auteur, quelques clichés de «l'autobiographeanalyste comme archéologue» (Poirier, 2001, p. 142). Le lecteur entre pas à pas dans cette dramatique politique. Comme l'écrit Poirier : " comme pour en finir avec le secret. [...] la psyché ressemble à des bandes dessinées ou à des affiches publicitaires, l'interprétation s'offre d'elle-même » (ibid., p. 146), de sorte qu'à la manière de Doubrovsky, le lecteur n'a aucune difficulté à collecter les clichés psychanalytiques, pensant être le premier spectateur de ce retournement robbegrillétien à l'égard de la psychanalyse. S'institue la dramatique politique du père et du fils à travers laquelle le psychanalyste incarne également une figure de nazi sadique devant lequel l'auteur, «[m]éticuleux, sadique, économe par surcroît » (Robbe-Grillet, 1984, p. 181), semble fuir avec ironie. Le fait de se présenter comme un "cas » clinique au même titre que Doubrovsky et que Guibert sur un plan biologique, participe de la stratégie de l'auteur en jouant sur le voyeurisme du lecteur : l'aveu n'en devient que plus précieux. 
Dans cette mise en scène, le lecteur semble être témoin de cet étonnant retournement par lequel soudain Robbe-Grillet serait contraint de reconnaître un inconscient à ses textes. Le jeu robbe-grillétien, utilisant la dramatique politique du fils et du père, est donc sensiblement différent du jeu doubrovskien. Robbe-Grillet fait trébucher le lecteur pour, à son tour, l'inciter à ce jeu. Quelques clichés psychanalytiques sont donc instillés puis éreintés. Gratuité dont la finalité est de servir d'appât au lecteur. À la différence de l'écriture de Doubrovsky ou de celle de Guibert, le lecteur est séduit mais davantage sur le mode du défi où il s'agit hystériquement de " [s]éduire pour mieux sévir » (Poirier, 2001, p. 150) et sous le couvert d'une épreuve de force. Ce n'est pas l'image du compagnon-lecteur qui prévaut mais celle de l'adversaire. Si Robbe-Grillet écrit contre lui-même, il écrit également « contre le public » $(1984$, p. 40), proposant quelques «leurres» (ibid., p. 41) mais également quelques «fables » qu'il nomme «opérateurs» (ibid. p. 18). Ces leurres seraient révélés dans la finalité de susciter le désir d'élucider les fables, le cadre de l'indécidable, bien sûr, agissant de manière tout aussi efficace. L'hystérie de l'autofictionnaliste agit à un autre degré que ce discours promouvant l'authenticité, cette écriture, le dévoilement du secret. L'autofictionnaliste ancre, précisément, cette stratégie politique cherchant à promouvoir par une rhétorique gauchiste une authenticité illégale, dans l'indécidable. Plus le discours de Don Juan apparaîtra dans cette authenticité propre aux révoltes d'un fils contre les mensonges patriarcaux, plus le caractère indécidable de sa promesse soutiendra le désir, c'est-à-dire le secret. La question du père et du fils est une dramatique politique servant à cette foi du lecteur en une élucidation libératrice mais, en un second temps, elle ne sert, par l'indécidable, qu'à un aveuglement ou à une opacification. 
L'autofictionnaliste défie hystériquement le lecteur. Il le convie à un ballet érotico-littéraire. Par ces petits aveux qui ne sont qu'appâts et qu'en somme nous pourrions remiser dans la catégorie des leurres, la dérision du procès autobiographique sert un jeu par lequel le lecteur est happé par sa pente naturelle. À partir de là, s'écrit l'indécidable. Il est donc une construction du secret, c'est-à-dire une fiction du secret, l'écriture du leurre n'étant qu'un préalable à l'écriture de l'indécidable, finalité ultime de l'autofiction. Valmont, Don Juan, Sade, avatars tutélaires, ponctuent l'angoisse de ne plus être «irrésistible » (Doubrovsky, 1994, p. 198). Cela «annihile» (ibid.). La construction du secret crée le désir du lecteur et c'est là le motif existentiel de l'autofictionnaliste. C'est par ce secret qu'il existe et non par l'aveu.

\section{Le compromis}

Nous sommes dans une logique du défi propre à Don Juan, à Valmont mais également à Sade. La représentation de la perversion est, dans l'autofiction, une métaphore de la perversion de la représentation (Scarpetta, 2001, p. 630). RobbeGrillet, comme Sade, instaure une loi pour mieux la transgresser. Le premier mouvement de ces deux auteurs serait sans doute de sécréter une règle morale « qui va fonder l'espace de son plaisir en même temps qu'elle l'interdit » (Robbe-Grillet, 2001, p. 230). Ainsi, Robbe-Grillet écrit, au début du Miroir qui revient, sans doute avec une certaine ironie: "je tâtonne encore ici, par perversité, dans l'entreprise réaliste, biographique et représentative » (1984, p. 30, je souligne). Tâtonner, c'est-à-dire sécréter l'entreprise réaliste, biographique et représentative 
pour mieux leurrer le lecteur avant que de s'abandonner au plaisir sadique de le délurer. La perversité tient à ce plaisir de renverser ou de mettre sens dessus dessous la situation, c'est-àdire au plaisir d'illustrer un modèle pour mieux le destituer. Robbe-Grillet évoque pour Sade «le viol du lecteur» (2001, p.100). Ne s'agit-il pas, invoquant au titre de la Nouvelle Autobiographie le programme du roman moderne, de « frustrer le lecteur, [de] l'appâter puis [de] le décevoir[; de] lui montrer sa place dans le texte [puis dans le même temps] l'en exclure » (Robbe-Grillet, 1984, p. 41) ? Le Miroir qui revient commence idéalement par cette promesse mensongère et séductrice : «Si j'ai bonne mémoire...» (ibid., p. 7) et s'achève par «Je ne me souviens de rien[.] Rien. Je ne retrouve rien.» (p. 219)

Doubrovsky, pour qualifier son écriture, évoque ce « "gynotexte" » (2010, p. 389) qui serait le jaillissement premier et spontané de l'écriture, puis le «"phallotexte" » (ibid.), un regard strict qui «surveille et corrige. Comme faisait mon père» (ibid., p. 390). En suivant ce chemin à l'allusion hystérique - ( $\mathrm{Tu}$ as un côté féminin, disait ma mère » (ibid., p. 389) -, l'autofiction serait un gynogenre face au phallogenre que représenterait l'autobiographie. Face au corps autobiographique entièrement soumis au regard comme à la prédication se dessine le corps autofictionnel pervers (en son sens étymologique) et résistant en sorte qu'il destitue ce regard en ouvrant une brèche semblable à la «reprise » kierkegaardienne telle que l'évoquent l'ouvrage éponyme de Robbe-Grillet ou l'article récent de Philippe Forest (2010, p. 132-133). La langue de Doubrovsky n'est pas seulement une déconstruction féminisante du Logos par le jeu feint de l'association libre, il est également une déconstruction féminisante du genre autobiographique. Comme un mobile à 
deux mouvements, le gynogenre autofictionnel n'existe qu'en miroir du phallogenre autobiographique. Si, dans l'autofiction, le sujet n'est plus comme dans l'autobiographie dans un rapport idolâtre d'imitation et d'adhésion au modèle mais, au contraire, dans un rapport de désintrication, l'un n'existe nullement sans l'autre. L'autofictionnaliste-fils ironisant de manière outrancière sur la soumission de l'autobiographe au modèle théorise et affirme une insoumission qui vaut confession. La figure fantasmatique du phallogenre autobiographique parcourt tout le corps autofictionnel. L'apologie autofictionnelle de la transgression, la "père-version » de l'autofictionnaliste, tend non seulement à maintenir l'impératif autobiographique mais, en le figurant sous les atours les plus totalitaires, à le radicaliser tout en le bafouant, et inversement. Le corps autobiographique est donc institué au cœur même de l'autofiction comme un corps légal et exemplaire à seule fin de subir les outrages sadiens d'une écriture perverse inversant les catégories réalistes et représentatives et transgressant les règles du genre sur le mode du déni, du leurre et de l'indécidable.

L'autofictionnaliste promet de se souvenir et de se dévoiler au lecteur, se référant aux codes autobiographiques d'une manière excessivement pointilleuse, sachant qu'il y échappera structurellement. Don Juan s'engage d'autant plus ardemment par les mots auprès d'une femme qu'il la sait interdite en sorte qu'il ne sera jamais mari mais toujours amant. Il est d'autant plus radical et excessif par le verbe - dépassant sans aucun doute le mari sur ce terrain - qu'il est bien certain de ne jamais avoir à en répondre dans la réalité. Le symptôme est là. Il faut tout à la fois se plaindre de son sort et, ispo facto, en jouir. Rivaliser avec le mari et tout à la fois s'assurer qu'il demeure bien en place pour ne pas avoir à s'y substituer. Se 
réclamer d'une castration plus haute dans les mots tout en s'assurant de ne jamais avoir à en répondre d'aucune dans la réalité. De même, il n'est jamais plus aisé à l'autofictionnaliste de faire une promesse ultra-autobiographique au lecteur en sachant que, sous couvert du pacte romanesque, il lui sera structurellement impossible de prendre la place de l'autobiographe. Mais comment ravirait-il une place qui justifie la sienne?

Affirmant échapper à tout compromis comme à tout compromission, l'autofictionnaliste construit, par l'entrée dans le pacte romanesque, la figure singulière d'un ultraautobiographe qui, s'instituant auto-analyste, fait le vœu d'une promesse plus authentique que celle de l'autobiographeanalysant tant qu'insoumis à tout regard comme à toute loi, il demeure au seuil de toute prédication. Or, la double figuration $\mathrm{du}$ père-rival en censeur-lejeunien et en analyste (aussi bien qu'en médecin) nous rappelle que la «père-version» autofictionnelle du genre autobiographique fait écho à toute une archéologie œdipienne se rejouant dans l'adultère. Tout entier dans l'inceste autofictionnel, l'autofictionnaliste-fils se tient au seuil de l'OEdipe autobiographique. De la sorte, il tient le jeu de l'énigme autant qu'il écrit en sorte qu'il ne dévoile pas mais au contraire ne cesse de soutenir le désir du lecteur. Il l'hystérise mais ressaisissant inlassablement son récit, tel Shéhérazade, il diffère cet aube annonciatrice de sa mort qui serait «Le dernier moi » tel que Doubrovsky affirme pouvoir nommer cet ultime livre dont le titre est en vérité Un homme de passage (Doubrovsky, 2010, p.385). Voici comment l'autofictionnaliste, feignant de critiquer cette propension de l'autobiographe à faire sa propre statue, construit la sienne de cette manière particulière qui n'est, au fond, pas exactement 
une légende puisque ce qui doit être lu demeure indécidable, mais un secret. L'auto-analyste autofictionnel ne vient pas en lieu et place de l'autobiographe-analysant mais rivalise avec lui comme l'hystérique avec le Maître. Il lui ravit avec brio le lecteur tout comme Don Juan ravit une femme à un mari ou à un père dont il ne désire nullement la place en une manière de compromis l'autorisant à jouir indéfiniment d'un Moi tenu au secret et sans cesse à re-conquérir comme à re-créer.

\section{Bibliographie}

DARRIEUSSECQ, Marie. (1996), «L'autofiction, un genre pas sérieux », Poétique, n 107, septembre, p. 369-380.

Doubrovky, Serge. (2010), «Le dernier moi», dans Claude Burgelin, Isabelle Grell et Roger-Yves Roche (dir.), Autofiction(s), Monts, P.U.L., p. 383-393.

—. (2006), « Claude Vigée, ou le mot de la vie (de L'Été indien à Moisson de Canaan) », dans Parcours critiques II (1959-1991), Grenoble, ELLUG, p. 47-51.

—. (2005 [1994]), L'Après-vivre, Paris, Grasset.

—. (2003 [1989]), Le Livre brisé, Paris, Gallimard, coll. « Folio ».

—. (2001 [1977]), Fils, Paris, Gallimard, coll. « Folio».

FOREST, Philippe. (2010), «Il faut continuer, je ne peux pas continuer, je vais continuer», dans Claude Burgelin, Isabelle Grell et Roger-Yves Roche (dir.), Autofiction(s), Monts, P.U.L., p. 127-144. 
GuiBert, Hervé. (2003 [2001]), Le Mausolée des amants, Paris, Gallimard, coll. « Folio ».

—. (1993 [1991]), Le Protocole compassionnel, Paris, Gallimard, coll. « Folio ».

—. (2005 [1990]), À l'ami qui ne m'a pas sauvé la vie, Paris, Gallimard, coll. « Folio ».

—. (1981), L'Image fantôme, Paris, Minuit.

Lejeune, Philippe. (1986), Moi aussi, Paris, Seuil, coll. «Poétique».

MEIGE, Henry. (1893), Le Juif errant à la Salpêtrière : étude sur certains voyageurs névropathes, Paris, L. Bataille.

POIRIER, Jacques. (2001), Les Écrivains français et la psychanalyse (1950-2000), Paris, L'Harmattan, coll. «L'CEuvre et la Psyché ».

Proust, Marcel. (1999 [1913]), " Du côté de chez Swann », dans À la recherche du temps perdu, Paris, Gallimard, coll. «Quarto ».

RobBe-GRILLET, Alain. (2003 [2001]), Le Voyageur, Paris, Seuil, coll. « Points ».

—. (1994), Les Derniers Jours de Corinthe, Paris, Minuit.

—. (1984), Le Miroir qui revient, Paris, Minuit.

SARTRE, Jean-Paul. (1969), "L'homme au magnétophone », Les Temps modernes, avril, $\mathrm{n}^{\circ} 274$, p. 1813-1819.

—. (1988 [1943]), L'Être et le Néant, Paris, Gallimard, coll. «Folio ». 
—. (2003 [1939]), "L'enfance d'un chef », dans Le Mur, Paris, Gallimard, coll. « Folio ».

SCARPETTA, Guy. (2001), "Représentation de la perversion et perversion de la représentation », Critique, août-septembre, $n^{\circ}$ 651-652, p. 630-639.

\section{Résumé}

Par l'entrée dans le pacte romanesque, l'autofictionnaliste affirme échapper à toute compromission comme à tout compromis. Il fait le vœu d'une promesse plus authentique que celle de l'autobiographe. Insoumis à tout regard comme à toute loi, il demeure au seuil de toute prédication. Ainsi, il construit la figure nouvelle d'un ultra-autobiographe qui, rivalisant avec l'autobiographe, lui ravit le lecteur exactement comme Don Juan ravit une femme ou une épouse à un père ou à mari dont il ne désire nullement la place en une manière de compromis.

\section{Abstract}

By adhering to the fictional pact, the autofictional writer argues that he can avoid compromising himself, hence rejecting any form of compromise. Immune to the law as to the human gaze, he vows that the promise he will make will be more authentic than the autobiographer's. As a result, he cannot be defined precisely. Through his actions, he constructs the new figure of an ultra-autobiographer who, by competing with the autobiographer, steals the reader from him as Don Juan would steal a woman or a wife from a father or a husband while rejecting any commitment - as if he made a compromise. 